Space 2014

4 - 7 August 2014, San Diego, California

\title{
Simulation/Emulation Techniques: Compressing Schedules With Parallel (HW/SW) Development
}

\author{
Mark L. Mangieri ${ }^{1}$ \\ June Hoang ${ }^{2}$ \\ NASA - Johnson Space Center, Houston, Texas, 77058 \\ e-mail: mark.l.mangieri@nasa.gov \\ e-mail: phuong-dung.t.hoang@nasa.gov
}

\begin{abstract}
NASA has always been in the business of balancing new technologies and techniques to achieve human space travel objectives.

NASA's Kedalion engineering analysis lab has been validating and using many contemporary avionics HW/SW development and integration techniques, which represent new paradigms to NASA's heritage culture. Kedalion has validated many of the Orion HW/SW engineering techniques borrowed from the adjacent commercial aircraft avionics solution space, inserting new techniques and skills into the Multi Purpose Crew Vehicle (MPCV) Orion program.

Using contemporary agile techniques, Commercial-off-the-shelf (COTS) products, early rapid prototyping, in-house expertise and tools, and extensive use of simulators and emulators, NASA has achieved cost effective paradigms that are currently serving the Orion program effectively. Elements of long lead custom hardware on the Orion program have necessitated early use of simulators and emulators in advance of deliverable hardware to achieve parallel design and development on a compressed schedule.
\end{abstract}

\section{Kedalion - NASA's Insight and SW Analysis Augmentation Lab}

Since Orion's inception, NASA has conducted a risk mitigation strategy by implementing an inhouse lab resource for targeted analysis and experimentation of Flight Test Article (FTA) and Orion concepts. Largely used for analysis and validation of Pad Abort-1 (PA-1) flight loads, and Guidance Navigation and Control (GN\&C) mode team support, Kedalion developed into a significant program resource recognized by Orion program management as an integral part of Orion's overall success.

Integration Synch Points (ISP's) are developed as interim demonstrations of Orion developmental technology at strategic points mutually agreed to by NASA and LM. These ISP's accomplish both milestones of progress and confidence building, especially in the area of inherently risky program technologies.

\footnotetext{
${ }^{1}$ Orion FSW V\&V and Kedalion Lead, Spacecraft Software Engineering Branch, Houston, TX 77058/MS ER6, AIAA Member.

${ }^{2}$ Kedalion Deputy, Spacecraft Software Engineering Branch, Houston, TX 77058/MS ER6.
} American Institute of Aeronautics and Astronautics 


\section{Kedalion's Purpose/Mission}

The Kedalion lab's fundamental mission is to provide a facility for risk mitigation throughout the life cycle of a space vehicle's development and operation. To achieve success the lab is oriented toward hands-on engineering analysis that allows NASA engineers to achieve a deeper understanding of the avionics software and hardware than could be achieved by only reviewing documentation. The lab is not, however, a formal oversight or verification facility but rather a flexible facility that welcomes collaboration with the vehicle contractors as well as personnel from other facilities to establish the best possible solutions to design issues, operational constraints and problem resolution as early in the life cycle of a vehicle as possible. It is this approach that allows NASA to blend years of experience on past programs with new ideas from industry and from within NASA to produce solutions that can guide a vehicle's development toward ultimate success.

\section{Multiple Fidelity Test Platforms}

The first test platform that a SW module is subject to test on the Orion program is the desktop SW development platform that every programmer has access to. This test platform includes all of the tools to design and develop SW algorithms, and a suite of simulation tools (SoftSim) capable of executing low fidelity executions of newly developed algorithms. SoftSim is an approach to bring target HW simulation to the developer's desktop SW development platform, based on contemporary advances in simulation technology.

Commercially known as the Honeywell (HI) Valfac Test Bench, the Vehicle Management Computer (VMC) Test Bench (VTB) platform was adapted first to successfully support the integration and testing of major SW subsystems on the FTA/PA-1 program. The Orion instantiation of the VTB is a modification of HI's commercial VTB concept to include support for Orion unique interfaces and target $\mathrm{HW}$, including Time Triggered Gigabit Ethernet (TTGbE) and VMC flight computers to support medium fidelity testing and integration.

The VTB platform is conceived as a highly adaptable and reconfigurable rig to support the many variations of tests required by the Orion SW project. The ultimate variation is the highest fidelity SW test platform known as the Super VTB. This test platform includes a dual string set of bus and target processors for testing redundancy and failover scenario's, as well as robust interface capability to access sensors/effectors allowing for first stages of subsystem vertical integration and test. 


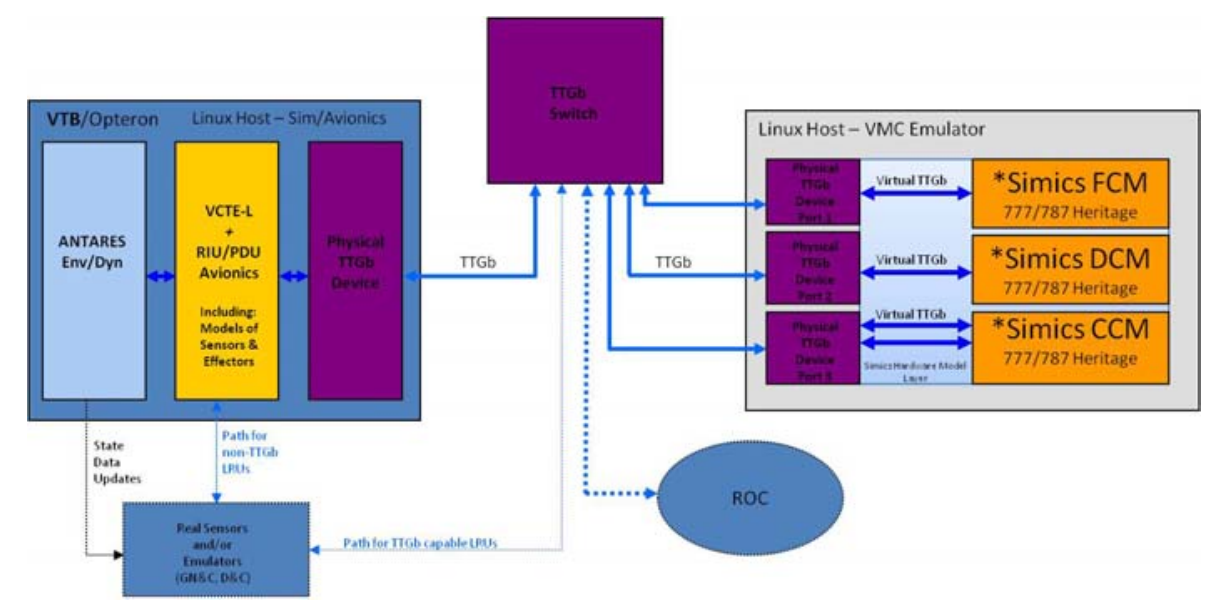

Early Kedalion Test Bed Concept with Simulated VMC

\section{Lab Architecture/Components}

The architecture of the Kedalion lab emphasizes flexibility, modularity and reconfiguration. The lab is populated with a variety of COTS hardware and software as well as some custom items and legacy assets from previous programs.

To establish a robust prototyping and development environment the lab is populated with a rich mix of development workstations that are powered by a variety of commercial computing operating systems including Microsoft Windows, Linux, IRIX and Solaris. By employing modern and legacy operating systems in the development environment engineers are able to maximize the use of modern tools as well as legacy software proven on prior programs.

More capable computing platforms are used to host simulation environments and emulation platforms. Embedded systems running real-time operating systems such as Green Hills Integrity and Windriver's VxWorks are used as needed to establish more robust real-time computing platforms. Non-real-time Linux platforms are also used for simulation and emulation, but through careful software design these computing systems can be used in integrated configurations with real-time hardware.

A variety of data bus configurations are also used to allow integration of a wide range of devices. These data bus types include MIL-STD-1553, commercial Ethernet, Time Triggered Ethernet, various serial interfaces and others as required. The concept of "bus adapters" is also used to allow legacy equipment to be mixed into configurations with more modern data busses. 
- Heavy use of COTS software tools

- IBM Rational Tool Suite

- Mathworks Model Based

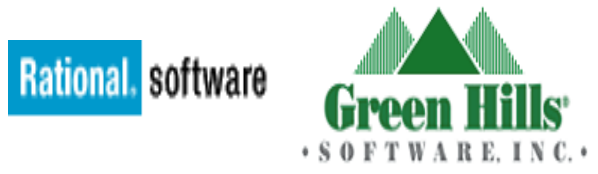

- NI Labview

- Windriver Simics \& VxWorks RTOS

- Green Hills Software - Multi, Integrity RTOS

- Reuse of common NASA developed tools

- Trick Simulation Framework

- EDGE - Graphics visualization package

- Multipurpose workstations and servers

- Multiple OS environment

- Linux, Windows, IRIX, Sun

- Diverse hardware platforms

- Orion flight computers

- Vehicle test bench

- Multiple I/O interfaces available

- Emulators and simulators used place of unavailable hardware

- Sensor integration platforms

- Rate Table for inertial sensor

- Star Field Stimulator for star trackers

- GPS signal generator for GPS

- Cockpit prototyping hardware

- Engineering quality Display Units - low cost

- Touch screen to virtualize display units

- Hand controllers

- "Out the window" views

\section{WIND RIVER}

Windows
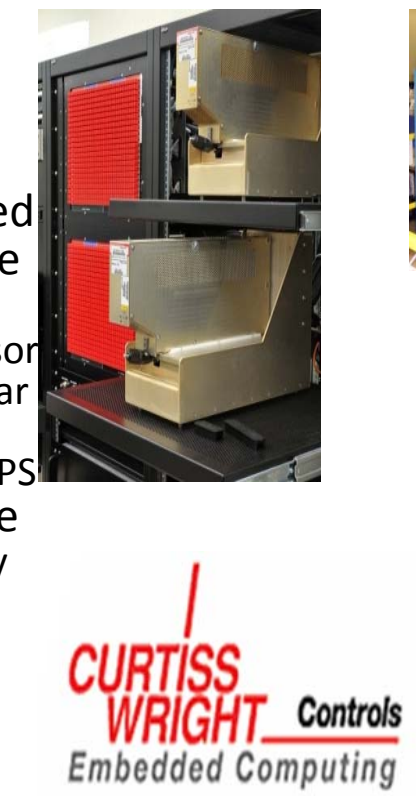

Embeded Computing

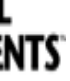
INSTRUMENTS
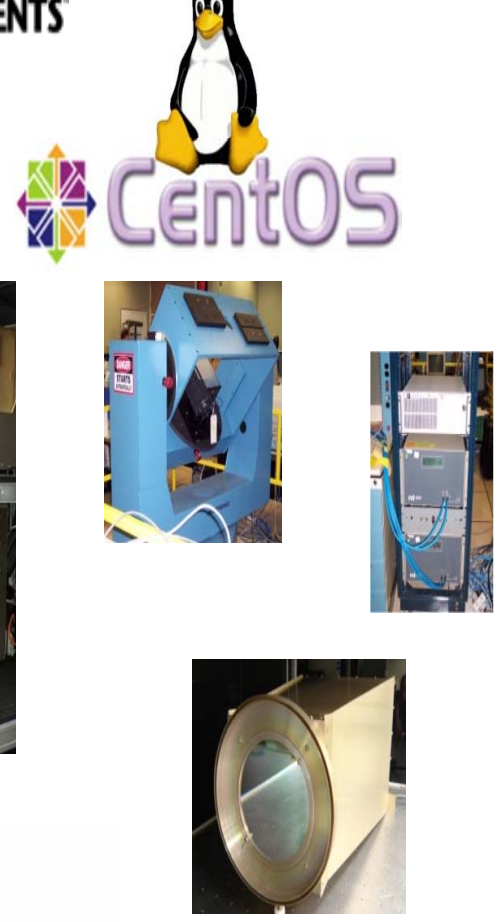

Kedalion “Tool Chain” and other Components 


\section{Software Tool Chain (Integrated SW Development Tool Set)}

Critical to understanding, designing and analyzing the avionics systems of a space vehicle is an understanding of the software tools used to build the vehicle's software products. For this purpose, the Kedalion lab has instantiated a modern and fully capable software development environment. While not limited to these tools the core of this development environment was based on the software tool chain selected by the prime contractor for the Orion program. For this reason, the IBM Rational Tool Suite was implemented as the foundation of the tool chain for the Kedalion lab. In addition, tools such as Mathworks' Matlab/Simulink, National Instruments Labview, Green Hills Multi, Microsoft Visual Studio, DiSTI's GL Studio as well as a variety of commercial software testing tools were added to the development environment to enhance the lab's tool chain.

\section{Sims/Emulators/HW/SW}

Another key feature of the Kedalion lab is the use of high fidelity simulations to add realism to test configurations. Vehicle avionics components are integrated with complex, high-fidelity simulations to produce a realistic configuration that can produce the same environmental and dynamic data as would be experienced in actual flight. Interactions with the natural environment and full vehicle dynamics as well as subsystem behaviors are produced through these complex, high-fidelity simulations.

Many of the models used in these simulations are reused common models and legacy models developed in other programs. This model reuse is enabled by the use of a NASA-developed simulation framework known as TRICK. The TRICK framework is used extensively in the Kedalion lab as the basis of most simulations and emulators. TRICK provides an extensive suite of simulation development, execution, monitoring and post execution analysis tools. In addition, TRICK provides a full-featured executive framework that can be configured and built into a variety of simulation architectures. TRICK provides auto-coding services that allow model developers to focus on their domain expertise rather than on the development of a simulation structure. This capability, combined with the easy reuse and reconfiguration of models, allows for very rapid development of complex simulations.

TRICK also provides simple options for integration with hardware interfaces and, therefore, provides an ideal framework for implementing emulators. If actual hardware elements are too expensive or simply not available an emulation of the device can quickly be developed through the use of TRICK.

\section{Emulating the Target}

Early versions of Orion hardware have become available to the project. Plans are in place to replace brass board flight computers with Engineering Development Units (EDU's). Flight 
software will be moved off the simulated or emulated platforms and onto flight computers. Since the changes to the configuration will happen at the hardware interface points the disruption to the operation of the test configuration will be minimal. As additional hardware becomes available it will be added to the configuration in a similar way.

In addition to "software only" simulations, such as the Simics based SoftSim, Kedalion personnel have pioneered higher fidelity emulated versions of the target computer. This hybrid solution between hardware and software provides an additional feature of providing a performance dimension not easily achieved with an exclusively software solution. Since the custom target is based on commercially available 750FX processor architecture, Kedalion was able to procure relatively inexpensive parts, and modify pre-existing NASA/JSC software, to quickly enable the production of the emulated target platform. The result of the Kedalion built emulation platform is a very high fidelity substitute for the vendors' brass boards, at a fraction of the cost.
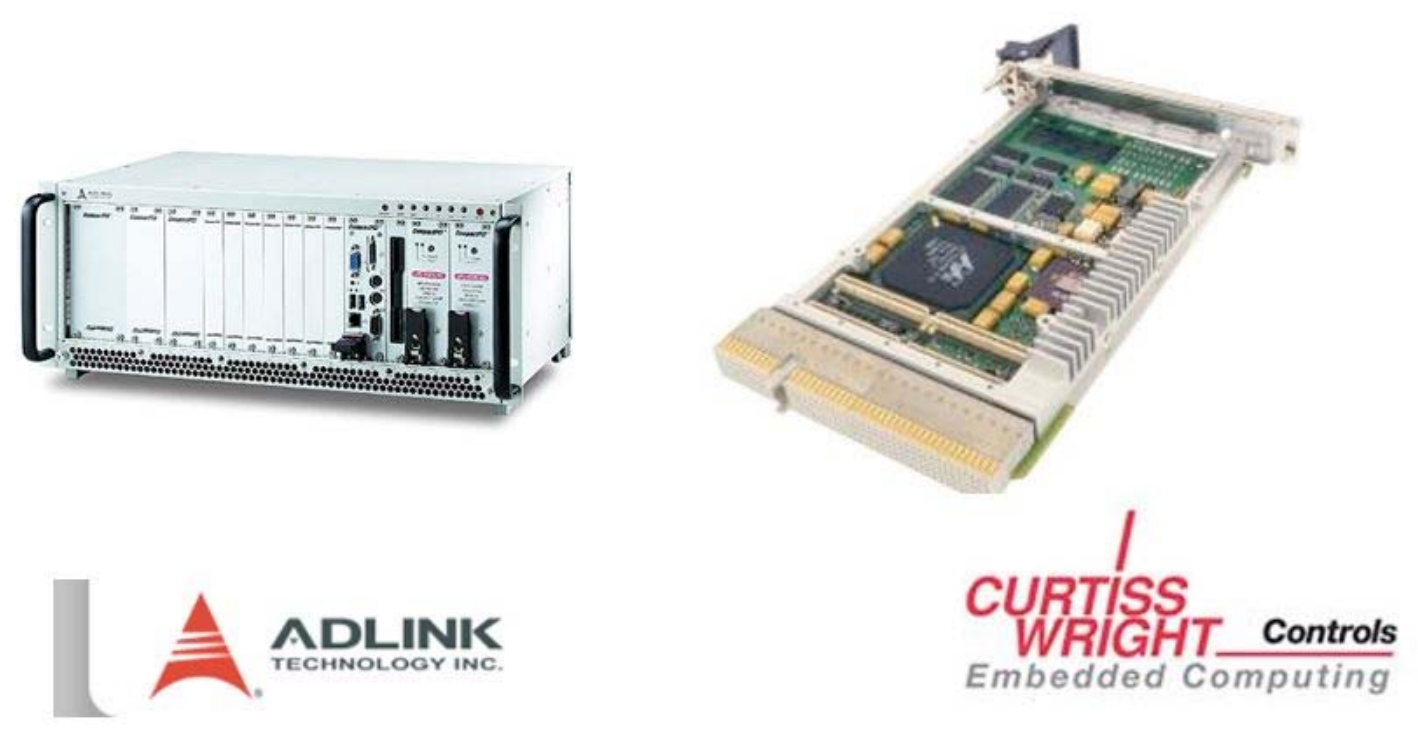

Commercial Hardware Emulation of Orion Flight Computer Brass Boards

\section{VMC Test Benches (VTB's)}

The VTB provides integration capabilities that allow flight software to be executed on actual flight processors while integrated with a full-featured simulation. A VTB allows scenario based testing to be performed where the flight software is executed in a "test like you fly" approach. Since the flight computers for Orion are based on Integrated Modular Avionics (IMA) architecture, a compatible test configuration is able to produce the fidelity necessary to reproduce actual flight conditions, as the flight computer would perceive them. The high fidelity simulation combined with a flight data bus capable 1/O pump makes this type of test 
configuration possible. The simulation feeds the I/O pump with all of the inputs necessary to fully populate the flight data bus.

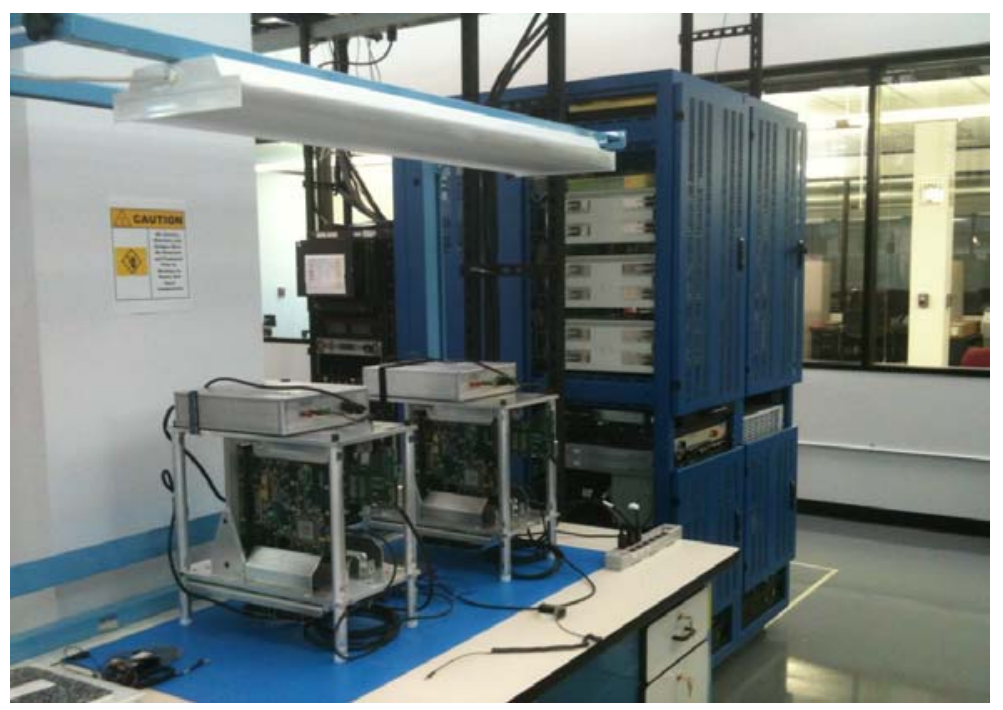

Kedalion built Test Bed with Honeywell Orion Flight Computer Brass Boards

\section{Current or Anticipated Orion Project Challenges}

In conclusion, the concepts described in this paper have enabled simulations and emulations to add flexibility to overall project cost and schedule. By substituting simulators and emulators early in the system development life cycle, extra parallelization is available as previously traditional task scheduling is considered. By identifying developmental lab environments within the program, emulators and simulators can serve as reasonable substitutes for the custom flight hardware, which is often very scarce and prohibitively expensive.

The Orion project anticipates some significant challenges and is open to collaboration and discussion with adjacent industries for solutions in these areas:

- Multi-lab connectivity issues (aggregated labs)

- IMA techniques and/or lessons learned

- IMA-based test bed strategies

- Simulation issues, specifically:

o Early target HW platform simulation (Windriver Simics or other)

o Environmental and/or HW simulation certification methods 


\section{Acknowledgments}

The authors thank the Orion project management team including David Petri for his guidance, collaboration, and support. The authors further thank the Spacecraft Software Engineering Branch management leads Steven Frederickson for encouragement and technical support in producing this paper. For support in sharing this information at the AIAA Space 2014 conference, the authors thank Rob Ambrose and Cliff Farmer, as well as the overall Orion project management team.

\section{References}

"Modular Verification: Testing a Subset of Integrated Modular Avionics in Isolation", Christopher B. Watkins, 25th Digital Avionics Systems Conference (DASC), Portland, Oregon, October 2006.

Feiler, Peter H. “Challenges in Validating Safety-Critical Embedded Systems.” Proceedings of SAE International AeroTech Congress. Seattle, WA (USA), November 2009.

https://www.sae.org/technical/papers/2009-01-3284

"ARINC 653 - An Avionics Standard for Safe, Partitioned Systems". WindRiver Systems/IEEE Seminar. August 2008. http://www.computersociety.it/wp-content/uploads/2008/08/ieee-cc-arinc653_final.pdf. Retrieved 2009-05-30.

RTCA, Incorporated, document RTCA/DO-178B, "Software Considerations in Airborne Systems and Equipment Certification,” December 1, 1992.

"How the IBM Rational Team Unifying Platform integrates with IBM Development Tools", IBM Corporation, Document G507-0962-00, July 2005

“Orion Flight Software V\&V and Kedalion Engineering Lab Oversight”, AIAA Space 2010, Document AIAA 20108761

“Kedalion: NASA’s Adaptable and Agile Hardware/Software Integration and Test Lab”, AIAA Space 2011, Document AIAA-2011-7176 